\title{
Tongue is the Face of the Malady? Differentiating in Desolate Distance: Locus -Tropical Field India
}

\section{Deepak Bhattacharya ${ }^{1^{\star}}$ and Pradhan PK $^{2}$}

${ }^{1}$ Head-Fight Malaria at Home, Kedar Gouri, Bhubaneswar, India

${ }^{2}$ Medical Officer-Red Cross Clinic, Collector cum District Magistrate, Koraput, India

\begin{abstract}
Koraput town, Odisha and the plateau peripheries is core malaria endemic region of India, Is well known for high drug resistance, year round obstinate manifestation and re-emergence of mutants. OMARIA (Odisha Malaria Research Indigenous Attempt) a field level effort (1997-2012) to Fight Malaria at Home led to a new anti-malaria. In dist., Koraput poly infestations with cross infections are quite common. There were no roads; no power; only natural stream water. Distances were covered on foot. Availability of pathology investigation tools and human resources were nonexistent. Therefore, neo ways and means were innovated to differentiate distinctions (clinic level) in such disconnected remote of the tropical-moist lush afforested rural India. Clinical conditions were varied and challenging. Observing the tongue and deciding the pathology, the diagnosis and the prognosis was attempted as choice tool (long period \{>decadal use multi-disciplinary team based approach).
\end{abstract}

Keywords: Omaria; Tongue observations; Buffy coat; Poly infections; Poly infestations

\section{Introduction}

Clinicians of yore used to take the condition of the tongue into consideration, which due the advent of diagnostic industrialized tools has fallen into disuse [1,2]. In present days clinicians normally do not take a visual examination into account. Few know the import and or the importance. Moreover, even in the past the visual appearance of the tongue and or the fossa was never used to make any assessment vis-à-vis malariasis and or concurrent infectionslinfestations. This report is based archived documents and does not constitute any 'clinical study' and or anything alike. It for the first time brings to light the fact that the appearance of the tongue can (possibly) spell the malady, especially malaria. Pan globally this is a first time report and specially weighs well due the large size of the sample, non-donor driven, non-target driven, and the long scope of the exercise. Among the historical ancient civilizations, that of India's continues only. She is credited with 1.2 billion citizens alongside drug resistant malaria and ravaging brain malaria. Bare foot, skimpily dressed sub-populations of tribal demography live in Koraput which is tropical, often breezeless, moist $\mid$ forest conditions thwart mosquito net use. High RH provides buoyancy. Vector bionomics gets easy (more during new moon and full moon weeks). Transmission gets to be continuous. This called for "Fight Malaria at Home", with a home makeable, hand makeable, rural medicine known as OMARIA' - Odisha Malaria Research Indigenous Attempt [2]. It is comprised of Ellagitanins + Ellagic acid with $\mathrm{K}^{+}$as anomer [3]. We report clinical aspects pertaining to the buffy coat on the tongue. Malaria combat operations with this India made novel drug source commenced in 1997, which attracted global attention [4-7]. There are also slimy coat', which is quite different and shall be dealt separately.

\section{Clinical Use Observations}

More than $20,000+$ cases were treated under "Fight Malaria at Home". These cases have generated a tome of observations which all have remained un-reported [8]. Due the absence of image recording devices and implements we have adopted schematic presentation and thereby limit the text to bare minimum.

\section{Only malaria}

Patients having only malaria and no other underlying infection or infestation presented a clear crimson tongue, (Figure 1). Untill these presents it was not known that malaria causes a neat and clean tongue.

\section{Malaria + typhoid}

Patients having malaria infestation and concurrent typhosa infection presented a tongue with a heavy buffy coat, (Figure 2). In malaria + typhoid cases, it is the non-brain malaria type symptoms that predominates (not very prominent). On application of OMARIA (or any anti-malarial) typhoid leaps to the fore, which erroneously gets labeled as 'brain malaria' - primarily because of the associated headache and delirium type symptoms. In such combined pathology, sweat is low-to-absent with obstinate-to-stiff headache at presentation; limited ambulation, acute mylgia, and asyclically alpine (restless; unable to sit). Post clearance of malaria, patient becomes supine and non-ambulatory (effect of typhoid, near morbid). If typhoid is cleared first the case then lapses into very severe malaria (pseudolidiopathic and often severe). Best results (synergic and side effect free) are indicated when OMARIA is followed by anti-bacterial therapy with a 4-8 hrs interval (not wider).

\section{Malaria + Renal Infection}

This leads the clinicians to question about urinary aspects. Relapse being the general feature inspite of loading and/or weak long OMARIA dosing. Conjoint application of Clotrimoxazole and OMARIA is very effective sans any side effect or contradiction (Figure 3 ).

\section{Malaria and URTI}

It is marked by least response to all variants of OMARIA. Alone antibiotics are effective. Albeit OMARIA seems to provide salutaryl

Corresponding author: Deepak Bhattacharya, Sri Radha Krishna, at Fight Malaria at Home, Kedar Gouri, Bhubaneswar, India , 751002, Tel: 8895997259 10674- 2430407; E-mail: oddisilab1@dataone.in

Received March 04, 2016; Accepted March 31, 2016; Published April 07, 2016

Citation: Bhattacharya D, Pradhan PK (2016) Tongue is the Face of the Malady? Differentiating in Desolate Distance: Locus -Tropical Field India. J Trop Dis 4: 211 doi:10.4172/2329-891X.1000211

Copyright: (C) 2016 Bhattacharya D, et al. This is an open-access article distributed under the terms of the Creative Commons Attribution License, which permits unrestricted use, distribution, and reproduction in any medium, provided the original author and source are credited. 


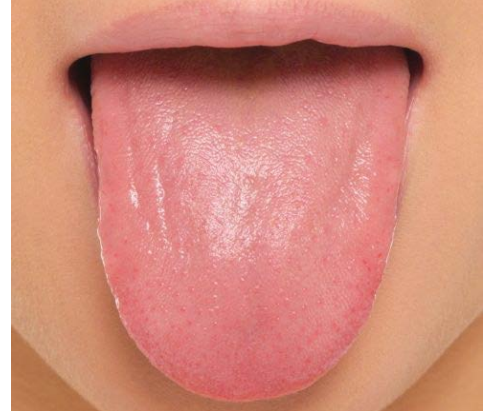

Figure 1: Infestation presented a clear crimson tongue.

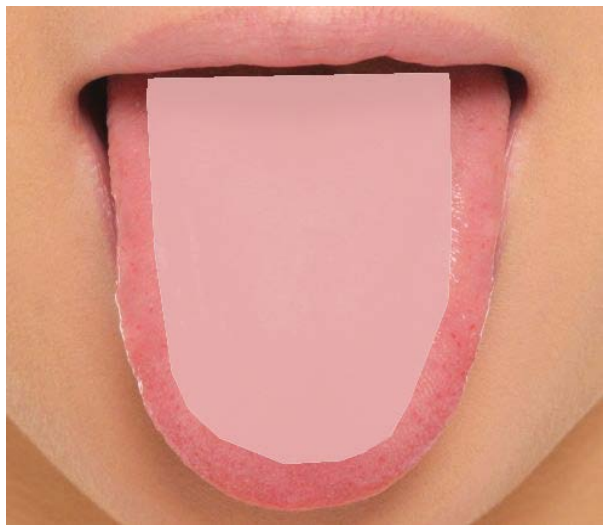

Figure 2: Part buffy coat is also noted during hepatic stage of the malaria parasite. Hence the patient is asymptomatic.

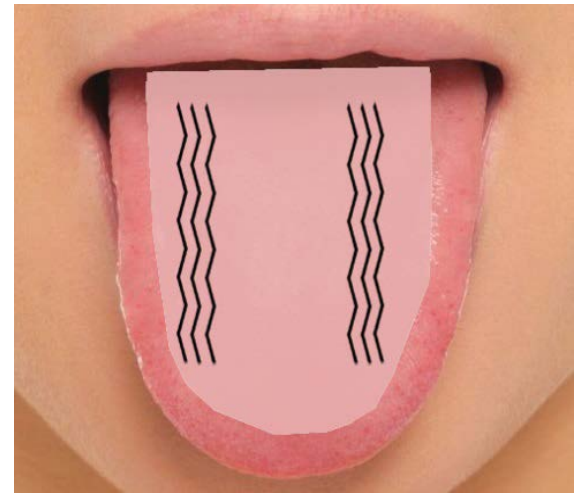

Figure 3: Patients having malaria + renal infection(s) presented a tongue with a buffy coat that have cracks at the sides.

buffer effect against side effects and intolerance to antibiotics and specially to multi-drug therapies (a practice became rampant in post OMARIA modality period). Until 2002 the term and concept of viral infection was not known in remote-rural Koraput. Patients having malaria + viral infection(s) presented a tongue with a buffy coat that is criss-crossed (figure not shown). Viral symptoms predominate at presentation, often having malaria like symptoms (resulting in clouding of symptoms and difficulty in diagnosis). Viruses being self-limiting, such symptom waned. These types respond very well to OMARIA. It was initially thought to be a variant of malaria (congruent symptoms).
Relief is precipitous when adjuncted with paracetamol (Figure 4). It transpired that Ellagitanins + Ellagic acid was also potent anti-viral having a very wide spectrum efficacy [9-11].

Patients having gastritis, epi-gastric, related clinical conditions and induced icterus present partial buff in the mid and inner tongue (tip portion being clean) with short cracks (supporting information).

The underlying ethos of such description (Sec. 2.5) is that in this type of buffy coat there is an area to area difference. Tongue is tough, tenacious; superbly blood flushed with micro-vessels and is mono tone...etc. It behaves and reacts as a single. Spatial segregation as in Sec. 2.5 indicates that the pathologies elicit variable responses on sectoral basis on the tongue. This means the tongue is effected in a manner about which we know little and have cared less. The discussions are illustrative about such thematic hypothesis. Tongue coatings may be mere stimulus based response. Or is it protecting self or something? Or are these coats have any therapeuticlprophylactic effect? What then is the etiology; the pathways; the stimulus?

\section{Discussions}

Due absence of optical recording devices and gadgets the naked observations were hand drawn as rough sketch with narratives in field note books (illustrations). Therefore, real time images are absent. Wherever, few are present, we are unable to present them because, we had thence not visulised the clinical import and the academic importance of hence had not taken informed consent for publication. Therefore, we have desisted reproducing them herein. Hemoparasystosis is a status wherein the non-motile, non-neuro sensitive i.e., neuro independent erythrocyte (which effectively is a sub organ, whole blood being the main) is the affected host. Hemoparasytosis is neither hepatic nor registers any effect on the digestive secretion mechanism. This is the cause of the clear tongue - is our considered view. These apart, malaria + gut infection is very common. Anti-protozoa and anti-helminthes medications are taken which all are chemo-therapeutics and logically also have anti-malarial effect. In this regard, we could not correlate reasonably because rural people also take numerous classical and natural medicines to control gut infections, as well (poly herbals that confound extensively). How much and which of the conventional and or the medicines are lost due to gut infestants posits neo opportunities.

Malaria + menopause geriatric cases indicated the most virulence in infection and obstinacy to clearance. These cases require longer or loading doses (OMARIA). Menopause geriatric persons are the grand old dotting nannies of the large loose Indian joint families (all religions/

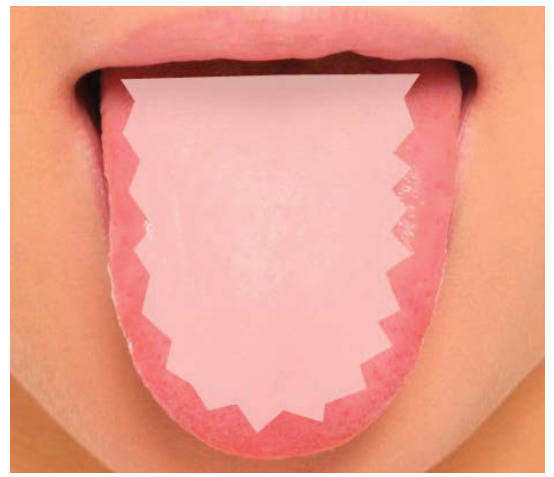

Figure 4: Patients having malaria + upper respiratory tract infection(s) present a tongue with a buffy coat that is crenated at the edges. 
regions). They abhor the mosquito net. Each such type presented tertian fever known as "pali jwara" (roster fever). This term connotes that a member of the joint family is sick at any point of time and it keeps rotating from individual to individual suggestive of low pathogenic mutant variety and $\backslash$ or mixed infestations). It also connotes that a particular geriatric male or female gets afflicted once every month, hence roster fever (pali jwara). This is suggestive of falciparum vivax (microscopically confirmed). We further noted that when left untreated and then shifted to uninfected families these geriatric-menopause pali jwara individuals seem to act as the springboard for infection (onto new members/guest/hosts) who all rapidly lapse into acute malaria. The from town visitor/guest, invariably. Often, gravitating into brain malaria status. And, all such alleged/conjectured transmission cases were quite resistant to CQ (intra muscular included). Families that had OMARIA treated menopause geriatric members presented no such cases, no such transmission. Same family, menarche females present the contrast. Our considered view is that the menopause geriatric nannies be the stock pool of the mutant types and also resistant Vivax. The Pali jwara male - females were singled out and given month long (free) sub-clinical OMARIA courses ( 1 cap OD, i.e., 30caps of $500 \mathrm{mg}$ each), with follow ups. It yielded results, quietly. OMARIA clears mutant-drug resistant hemoprotozoa and blocks its transmission (clinically sure). Since the menarche group presents a diametrically contrast it is conjectured that menarche (primed menstrual state) may thwart hemoparasytosis in spite of anabol.

It is interesting to note that in the rural country side the buffy coat returns to variable degrees in neat/only malaria infestation cases (as in F-1) post clearance with OMARIA. Such re-manifestation is not immediate post the wane of malariasis, but a week post OMARIA modality. This is primarily because dental oral care is scant-to-nil. The coat returns abruptly with CQ and ACTs (Artimisinin Combined Therapies) and is delayed if CQ and ACTs be adjuncted with OMARIA. With loading dose of the CQ or ACTs and multi drug therapies (MDT) such coat persists. In all other parasitemias, infections and medications thereof, the buffy coat clears post therapy (distinction). This portends as that the CQ; ACT and MDTs have toxic effect on hepatocytes, gut and the nephrons apart from inducing tachardia and hypotension (OMARIA has no such side effects or contradictions). Culturally, and historically rural India use fresh twigs and wood ashes as tooth brush and scrub material, respectively. Such dental scrubbing twigs are alkaloid rich which in turn are potently lytic for pathogens and non-pathogens of the mouth, while they up-regulate blood flow in micro-vessels of the scrubbed lamina and the fossa. The ashes are sterile, neutral and absorbent cum binders. Tooth and gum scrubbing in a mixed manner with Neem; Saal; Sahada; Pijulee (Azardica, Treblus; Guava) and ashes becauses a clear fossa, neat tongue and tight gums. All this also offers additional scope for study. Hemoparasytosis is a malady of the RBCs (which is an independent sub-organ; the sole non motile cum moor-less cell system; which also do not form any continuous cell line, remains segregated always in dynamic motion (inertia), which is why, body's defense mechanism is unable to mount any attack (neither any is oriented, biochemically). Instead, (in status malariasis $\backslash$ filariasis) various epitheliums and fixed cell lines are insulted by the body's defense system. As a result multi centric, systemic-innate inflammation cascades are triggered. On the other hand, the malarial (hemo) parasites at hepatic phase are sans any toxicity i.e., no hepato-toxicity thus there is no buffy coat (clear tongue; F-1). It is associated with toxicity, including hemozoin (crystalline, stable and toxic lipid rich ferriprotoporphyrin IX) Hence, hepatotoxic and or hepatogenic moieties are uncalled for as therapeutic candidates. Inter alia this also suggests that bacterimia, viremia and even drug side effects / contradictions are maladies that work via/upon the all other cell lines other than the RBCs or the plasmodium/parasites. They because hepatotoxicity with the metabolites that are expressed, which in turn gets reflected on to the human tongue. Hence there is a need to know more.

\section{Conclusion}

Examining the protruded tongue also concurrently results in an auto examination of the fossa the epithelium of which is very sensitive. Hence, visual examination is quite suggestive (especially for initiating an intervention therapy until the diagnostics results arrive). Malaria and typhoid are galloping in nature with grave prognosis. Clinicians cannot wait. In the instance study, most of the cases until almost 2010 presented having not been pre-medicated. Therefore we feel confounding is least. Tongue-and -fossa examination is also of much help (indicative) even in the most sophisticated clinical-hospital settings. In concurrent infection + infestation (i.e., bacteria/virus + hemoprotozoa) either play down the pathogenic virulence of the other (inter member limiting effect of poly pathogenic infections/infestations). A buffy coat leads to a reduction in appetite with an up-regulation in the expression of the digestive enzymes from the maxillofacial glands. Such enzymes jointly with the 'twig alkaloids' are lytic for cellular pathogens and neutralising effect on the expressed toxins. While the expressed enzymes help the pancreas in its endocrine function. The human tongue offers (possible) scope for neo diagnostics (non-invasive vis-à-vis infestation, infections and inflammation). Prima-facie, tongue is the face of the malady.

\section{Acknowledgement}

The Addl. Dist Magistrates (DM); The Collector cum DMs, all along facilitated this decadal+ study and provided on ground support. Special mention is for Mr. B. M. Bhuyan (Administrative Service-I), the Secretary, Koraput, District Red Cross Society cum The Dist., Emergency Officer (all of the Collectorate) for providing yeoman support to Fight Malaria at Home - OMARIA.

\section{Conflict of Interests}

There is no Conflict of interests.

\section{References}

1. Oxford Text Book of Medicine. In: David AW, Timothy MC, John DF (eds.) London.

2. Deepak B (2015) A New Natural Anti-Malaria Source India. J Trop Dis 4: 2-4

3. Deepak B, Bhuyan BM, Pradhan PK, Nayak DK (2013) Transmission Blocking of Year Round Resistant Malaria in Koraput (India) by OMARIA- A New Antimalarial Phytotherapy. British Journal of Pharmaceutical Research 3: 54 57.

4. Patnaik N (2000) BBC: India claims malaria cure.

5. Bhattacharya D (2004) Punica granatum's dermis indicates prophylaxis against malaria \& wide spectrum anti-viral property in human use 171: 288.

6. Economic Times (2000) “Herbal Anti-Malaria Drug on Anvil, News Report”. India's National Circulation-News Paper

7. Deepak B (2006) Fight Malaria at Home, Express Pharma. Fortnightly Insight for Pharma Professionals.

8. Deepak B (2011) Fight malaria at home: Therapeutic and prophylaxis clinical data Asian Pacific Journal of Tropical Diseases 1: 142-149.

9. National Institute of Virology Pune. Annual Report 2002-2003.

10. Bhattacharya D (2007) Tannins, Ions, Cations \& Malariasis: Observations \& Theory. American Journal of Tropical Medicine and Hygiene 77: 27.

11. Dell'Agli M, Galli GV, Corbett Y, Taramelli D, Lucantoni L, et al. (2009)Antiplasmodial activity of Punica granatum L. fruit rind. J Ethnopharmacol 125: 279-285. 